\title{
1 Genome evolution in a putatively asexual wasp
}

3 Eric S. Tvedte ${ }^{1,2}$, Austin C. Ward ${ }^{1}$, Benjamin Trendle ${ }^{1}$, Andrew A. Forbes ${ }^{1 *}$,

4 John M. Logsdon Jr. ${ }^{*}$

5

$6{ }^{1}$ University of Iowa, Department of Biology, 143 Biology Building, Iowa City, IA, USA.

$7{ }^{2}$ Current address: Institute for Genome Sciences, University of Maryland School of Medicine,

8 Baltimore, MD, USA.

9

$10 *$ Corresponding Authors: john-logsdon@uiowa.edu or andrew-forbes@,uiowa.edu

11

12 Keywords: Sex, Diachasma, Mutation

13 


\section{Abstract}

15 Asexual lineages are destined for extinction — a result predicted by theory and revealed in

16 practice. Short-term benefits of asexuality are eventually outstripped by their fitness costs: losses

17 of sex and recombination are together expected to reduce efficacy of selection, increase mutation

18 load, and thus, lower fitness. We characterized genomic patterns of accumulating mutations in

19 Diachasma muliebre, a parasitic wasp that has apparently lost sex, an inference supported by

20 many decades of field collections of 1000s of individuals in which only females were found. The

21 split between D. muliebre and its closest sexual relative, Diachasma ferrugineum, is quite recent,

22 allowing us to observe initial events in the evolution of this putative asexual species. First, we

23 find a faster rate of molecular evolution across the $D$. muliebre genome. In addition, we observed

24 a marked excess of replacement nucleotide substitutions in orthologous genes in the putatively

25 asexual $D$. muliebre when compared to $D$. ferrugineum. This pattern directly indicates genome-

26 wide relaxed selection in this young, putatively asexual species, the resulting mutational load

27 from which is expected to ultimately lead to extinction. However, these genomic effects occur in

28 the presence of genomic recombination initially detected by a previous study and also supported

29 by analyses of genome-wide substitution rates within codons. In addition, following completion

30 of the genome sequence and its analysis, we discovered two $D$. muliebre males, suggesting the

31 possibility of rare sex in this species. Haplodiploid animals, including the sexual ancestors of $D$.

32 muliebre, bear small genetic loads, likely making their initial transitions to asexuality relatively

33 benign. Paradoxically, an elevated rate of mutation accumulation resulting from asexuality, when

34 accompanied by retention of recombination and/or rare sex, could actually be beneficial: we

35 hypothesize that the novel variation introduced by mutation along with limited shuffling of genes

36 may facilitate initial adaptation and extend persistence of such lineages. 


\section{Introduction}

38 Sexual reproduction is omnipresent among eukaryotes despite its considerable metabolic and

39 genetic costs. This apparent paradox has generated substantial discourse regarding the presumed

40 benefits of sex (Otto 2009; Hartfield and Keightley 2012). The ubiquity of sex and the "twiggy"

41 representation of asexual lineages on phylogenetic trees have been interpreted as signals that

42 transitions to asexuality are always accompanied by major fitness costs — and that asexuality is

43 eventually an evolutionary dead end (Maynard Smith 1978; Bell 1982).

44 Explanations of why fitness would decrease after a transition to asexuality usually invoke

45 the increase in genetic load of deleterious mutations in asexual genomes (Lynch et al. 1993).

46 Without outcrossing and meiotic recombination, asexuals are predicted to experience limitations

47 on the efficacy of selection to act independently within and among loci and to expunge

48 deleterious alleles (Hill and Robertson 1966; Felsenstein 1974). Moreover, asexual individuals

49 with low genetic loads may often be lost via drift and the inability to restore these least loaded

50 genotypes via recombination should lead to the irreversible accumulation of deleterious

51 mutations within an asexual lineage (Muller 1964; Charlesworth and Charlesworth 1997).

52 Overall, high genetic loads experienced by asexual lineages is expected to limit their ability to

53 persist over long evolutionary timespans.

The absence of meiotic recombination and the resulting reductions in efficacy of

55 selection in asexuals is predicted to produce a distinct genomic signal: a genome-wide, increased

56 ratio of nonsynonymous to synonymous differences between species (Glémin and Galtier 2012).

57 Previous studies provide some support for a faster accumulation of putatively deleterious

58 mutations in asexuals; however, until recently most have examined just a small number of genes,

59 often rendering the results equivocal (Hartfield 2016). Recent transcriptome and whole-genome 
60 analyses have improved the amount of data bearing on this question, but have also shown mixed

61 support for increased mutation accumulation in asexual lineages (Jaron et al. 2020). Some

62 studies have suggested a reduced efficacy of purifying selection to remove harmful mutations in

63 clonal asexual lineages (Hollister et al. 2014; Lovell et al. 2017; Bast et al. 2018), while others

64 show no effect (Ament-Velásquez et al. 2016; Lindsey et al. 2018; Brandt et al. 2019) or even

65 more effective selection in asexuals (Brandt et al. 2017). [It should be noted that we use

66 "mutation accumulation" to describe differences between species in this context instead the

67 cumbersome, but more accurate, "substitution accumulation”.]

68 Why have previous studies not detected a consistent mutational signal in asexual

69 genomes, if it exists? Comparisons between sexual and asexual sister species are difficult due to

70 uncertainties about the age of the split (Ament-Velásquez et al. 2016; Brandt et al. 2019) (and,

71 for comparisons among asexual groups, variability in asexual age), or they are complicated by

72 hybridization and/or polyploidy in the transition event (Hollister et al. 2014; Ament-Velásquez et

73 al. 2016). It is also possible that some presumed asexuals are, in fact, not entirely asexual, but

74 instead are capable of sexual reproduction, albeit occasionally, or even rarely (Schurko et al.

75 2009). Nonetheless, a number of genomes from presumed asexuals have been sequenced and

76 compared to their sexual relatives, as summarized recently (Jaron et al. 2020). Notably, no

77 consistent feature — including mutation accumulation — is shared among all (or even a majority)

78 of the 24 presumptive asexual animal genomes that Jaron et al. (2020) surveyed. Some other

79 unusual features found among some asexual animal genomes include changes in heterozygosity,

80 increased numbers of horizontally transferred genes, reductions in transposable element content,

81 and loss of genes involved in sexual reproduction. Although these data may indicate no feature

82 shared in common (and diagnostic for) asexual genomes studied to date, many of these odd 
83 aspects are predicted to occur only following the long-term persistence of asexuality. Indeed, it is

84 possible that comparisons among asexual genomes with different divergence times from their

85 sexual ancestors obscures potential genomic commonalities, in particular those that might arise

86 either in late or early stages of asexuality.

An ideal system to assess the initial impacts of asexuality on a genome would be one in

88 which the transition to asexuality is recent, where the ecology is little changed, and for which

89 genomic data are readily available for evolutionary study (Tvedte et al. 2019a). The presumed

90 asexual parasitic wasp Diachasma muliebre split from its sexual relative Diachasma ferrugineum

91 in an apparent single loss-of-sex event 10,000YA-1MYA (Forbes et al. 2013)(Supplemental

92 Information), but the two wasps otherwise differ very little, attacking sister species of specialist

93 tephritid fruit flies on cherry trees. Although details of the D. muliebre reproductive life cycle are

94 essentially unknown, separate lines of evidence are consistent with it being asexual, including: a)

95 in $>2300$ individual specimens from $\sim 70$ years of field collections and across its entire

96 geographic range, only females have been collected and not a single male was identified prior to

97 this work (see Results \& Discussion and Supplemental Information; Table S1) — though field

98 collections analyzed in 2020 revealed the presence of two apparent $D$. muliebre males and b)

99 there appears to be complete association between mitochondrial and nuclear genotypes (Forbes

100 et al. 2013).

101 The loss of sexual reproduction has both short- and long-term consequences which may

102 be revealed in the genomes of asexual lineages. Here, our comparative genomic analyses of the

103 putative asexual wasp D. muliebre and its close sexual relative, D. ferrugineum show striking

104 patterns of sequence divergence associated with a recent transition to asexuality and consistent 
bioRxiv preprint doi: https://doi.org/10.1101/2020.12.23.424202; this version posted December 24, 2020. The copyright holder for this preprint (which was not certified by peer review) is the author/funder. All rights reserved. No reuse allowed without permission.

105 with current evolutionary theory. Notably, these patterns emerge and persist even in the presence

106 of detectable genomic recombination and the presence of rare males in this species.

107 


\section{Results \& Discussion}

109 We obtained Illumina sequencing datasets from the genomes of both $D$. muliebre and $D$.

110 ferrugineum (Table S2) and compared these with existing genome sequences from their next

111 closest sexual relative (Dichasma alloeum) and two other sexual braconid wasps (Fopius

112 arisanus, Microplitis demolitor). After considerable annotation and filtering (see Materials \&

113 Methods), we recovered 3,127 genes with full coding DNA sequences (CDS) coverage in all

114 five wasp species, representing $\sim 25 \%$ of the predicted genes in the $D$. alloeum genome (Tvedte 115 et al. 2019b).

116 In the absence (or decreased functionality) of sex and canonical recombination in 117 asexuals, the proportion of mutation-free genes is predicted to decrease and, by inference, their 118 mutational load will increase. Consistent with this prediction, we find that gene sequences from 119 D. muliebre are more different than genes from D. ferrugineum when both are compared to $D$.

120 alloeum. Indeed, 1,219 of the 3,127 genes have higher pairwise distances in D. muliebre

121 (pdistDM > pdistDF), while 899 genes have higher pairwise distances in D. ferrugineum

122 (pdistDM $<$ pdistDF) (Figure 1; $\left.\chi^{2}=48.35, \mathrm{p}<0.0001\right)$. Additionally, 149 genes are identical

123 between $D$. ferrugineum and D. alloeum (pdistDF $=0$ ), compared to 133 identical genes between

124 D. muliebre and D. alloeum (pdistDM =0).

125 Despite the recent origin of this asexual wasp, comparisons of pairwise distances across

126 the entire gene set display a prominent shift of the $D$. muliebre mutation landscape relative to $D$.

127 ferrugineum, reflecting a faster rate (accumulation) of change in the asexual (Figure 2, Figure

128 S1). There is a significant difference between mean pairwise distances for $D$. muliebre genes

129 (MpdistDM $=0.005496, \mathrm{SD}=9.365 \mathrm{E}-6)$ relative to $D$. ferrugineum genes $(\mathrm{MpdistDF}=$

$130 \quad 0.005089, \mathrm{SD}=7.953 \mathrm{E}-6$ ) when compared to $D$. alloeum homologs; this indicates more new 
131 mutations persist in the asexual lineage (Wilcoxon rank sum $p=4.17 \mathrm{E}-7$ ). Although the distinct

132 mutational landscapes in sexual and asexual wasps could be confounded by the non-

133 independence of genes contained in linkage groups, the sampled scaffolds did not show evidence

134 of substantial linkage among genes in our dataset (Supplemental Information).

To further evaluate patterns of mutation accumulation in Diachasma genomes, we

136 combined coding sequences from all 3,127 genes into a concatenated dataset. Given the recency

137 of the presumed loss-of-sex event in these wasps, the signal generated by differences in rates of

138 mutation accumulation is likely to still be small, and therefore comparisons involving single or

139 few genes may not be informative (Tvedte et al. 2017). A maximum likelihood analysis of the

140 concatenated coding sequences displays a clear increase in the overall rate of molecular

141 evolution in D. muliebre relative to D. ferrugineum (Figure 3A). Indeed, the total number of

142 unique differences in D. muliebre is greater than in D. ferrugineum $\left(\chi^{2}=256.91, \mathrm{p}<0.0001\right)$

143 consistent with observations from pairwise distance data (Table 1).

144 To distinguish between effects generated by accelerated mutation rate per se in the

145 asexual and reduced efficacy of selection, we also evaluated these combined datasets by codon

146 position. An increase in the intrinsic mutation rate in an asexual lineage would be characterized

147 by a greater number of mutational changes at selectively neutral sites (e.g. synonymous sites).

148 We analyzed the aligned $3^{\text {rd }}$ codon positions to estimate this effect. Similarly, if asexuality is

149 associated with the reduction in the efficacy of selection to act independently on physically

150 proximate loci to remove mildly deleterious mutations, we expect to observe the increased

151 incidence of substitutions at nonsynonymous sites, estimated here using $1^{\text {st }}$ and $2^{\text {nd }}$ codon

152 positions. As a result of this increased rate of mutation in the asexual wasps, D. muliebre shows a

153 longer branch length relative to D. ferrugineum when using $3^{\text {rd }}$ codon position partitions, and a 
154 relative rate test indicated a significant excess of unique differences in D. muliebre $\left(\chi^{2}=142.76\right.$,

$155 \mathrm{p}<0.00001)$ (Table 1, Figure 3B). Similar to $3^{\text {rd }}$ codon position observations, D. muliebre had

156 longer branch lengths compared to those of its closest sexual relative, D. ferrugineum (Figure

157 3C). The number of unique differences was substantially higher in $D$. muliebre at $1^{\text {st }} / 2^{\text {nd }}$

158 positions $\left(\chi^{2}=123.24, \mathrm{p}<0.00001\right)$, and the asexual genome also contained a larger proportion

159 of total unique differences occurring at $1^{\text {st }} / 2^{\text {nd }}$ codon positions (Table 1). We tested for selective

160 pressures on synonymous sites by measuring the effective number of codons (ENC) and codon

161 deviation coefficients (CDC) and our results suggest that accelerated evolution in D. muliebre is

162 not explained by relaxed selection on codon usage (Table S3, Supplemental Information).

163 Taken together, this pattern suggests that D. muliebre possesses a larger mutational load relative

164 to $D$. ferrugineum, with putatively deleterious mutations more often retained in the asexual

165 lineage.

166 To further consider the possible effects of asexuality on individual genes, we extended

167 our analyses to compare the rates of molecular evolution between $D$. muliebre and $D$.

168 ferrugineum within and among each of the 3,127 genes. Our comparisons of the amino-acid

169 replacement (nonsynonymous) rates of substitution $(\mathrm{dN})$ between genes revealed results that are

170 mutually complementary. First, there are significantly more genes in the zero class of estimated

171 replacement substitutions in the sexual species (D. ferrugineum) compared to the asexual

172 (Figure 4). Second, there are significantly more genes having replacement substitutions $\left(\chi^{2}=\right.$

$1735.303, \mathrm{p}=0.0203)$ in the asexual (D. muliebre) compared to the sexual species. Third, there are

174 significantly more replacement substitutions (estimated by $1^{\text {st }}$ and $2^{\text {nd }}$ position differences) in $D$.

175 muliebre $\left(\chi^{2}=33.006, \mathrm{p}<0.0001\right)$, even when correcting for the fact that there are more overall

176 differences in D. muliebre $\left(\chi^{2}=4.476, \mathrm{p}=0.0344\right)$. Strikingly, the pattern in Figure 4 shows a 
177 clear shift, further indicating reduction in the efficacy of selection in the asexual. Our parallel

178 analyses of synonymous (dS), did not reveal a significant pattern of differences between the

179 sexual and asexual, beyond an overall excess seen in D. muliebre-which is expected given that

180 D. muliebre has more overall substitutions (Figure S2, Supplemental Information). These

181 results provide a clear snapshot of the initial processes of molecular evolution near the onset of

182 asexuality: genes that are evolving under varying degrees of purifying selection-from the

183 strongest to the weakest - show an increased rate of replacement substitution in the asexual. This

184 effect appears to be general and genome-wide; however, further analyses will be needed to

185 assess this among and between genes in various functional classes.

In addition to the overall increase in the rate of molecular evolution resulting from

187 reduced selection in an asexual, both the efficacy and pattern of selection will be drastically

188 altered across the genome. In fact, for asexuals that are not experiencing recombination, their

189 entire genome is inherited clonally, disallowing mutations in adjacent sites in the genome to be

190 selected independently — even if their selective effects differ. Thus, in asexuals, substitutions at

191 silent versus non-silent codon positions would not directly follow the familiar patterns observed

192 in sexual species. Given that rates of new mutations at $1^{\text {st }}, 2^{\text {nd }}$ and $3^{\text {rd }}$ positions in codons are

193 approximately equal within a species, their rates of substitution after the onset of clonality would

194 only reflect the removal of deleterious mutations along with all linked mutations (on that

195 chromosome). Then, the prediction in a clonal asexual will be an increase in the observed ratio of

196 non-silent: silent codon substitutions across the genome relative to the ancestral sexual state.

197 However, since recombination has previously been inferred in D. muliebre (Forbes et al. 2013;

198 Tvedte et al. 2017) — a result which may be related to the presence of rare males (see below) -

199 the prediction is that substitution patterns within codons in this asexual should be more similar to 
patterns in sexuals than to a true clonal asexual. In fact, the ratio of observed substitutions at $1^{\text {st }}+$

$2012^{\text {nd }}$ positions versus $3^{\text {rd }}$ in D. muliebre is 0.42 , compared to 0.37 in D. ferrugineum (Table 1).

202 These data further indicate that D. muliebre is experiencing a measurable decrease in the

203 genome-wide efficacy of purifying selection (on the order of $\sim 15 \%$ ), discounting possible effects

204 of positive selection (which would be predicted, on average, to be similar in both species). When

205 comparing the inferred synonymous versus non-synonymous rates across all 3,127 genes, the

206 mean dN minus mean dS is $20 \%$ greater in D. muliebre (data not shown), consistent with the

207 observed patterns by codon position. Notably, the similarity of this ratio of observed

208 substitutions between the sexual and asexual wasps is consistent with the presence of

209 recombination in $D$. muliebre (where the extreme predicted ratio in a clonal asexual with no

210 recombination should approach 2.0); however, the generality of this result is unknown, as we are

211 unaware of other such analyses in the literature. Two major hypotheses could explain why

212 substitution rates within codons vary somewhat independently, albeit less so than for a sexual: 1)

213 Perhaps D. muliebre is not, in fact, strictly asexual. The recent discovery of rare males may

214 occasionally provide opportunity for infrequent sex and meiotic recombination; 2) Sufficient

215 meiotic recombination could be provided by automictic reproduction in D. muliebre. Prior to the

216 recent discovery of males, we favored the second hypothesis given the data currently available

217 (Forbes et al. 2013; Tvedte et al. 2017); however, this will require further investigation,

218 especially focusing on the biological relevance of $D$. muliebre males (see below and

\section{Supplemental Information).}

220 In any case, the clear result of increased rate of genome-wide substitution patterns we

221 observe in D. muliebre are themselves strong predictors for asexuality and are not consistent

222 with sex. Nonetheless, the recent discovery of two D. muliebre males in 2020 field collections 
223 has required us to consider possible implications for asexuality in this species. First, given the

224 extreme rarity of finding males in this species (only 2 males in over 2800 wasps collected over

$225 \sim 70$ years), it is likely that the vast majority of reproduction in this species is asexual. It is

226 possible that these are an example of "spanandric", non-functional, males that are rarely

227 produced in some otherwise obligately asexual species (Smith et al. 2006). Alternatively, these

228 males could be functional and only rarely participate in sexual reproduction in this species.

229 Although new questions are raised (see Supplemental Information) and additional analyses

230 will be required to definitively address the possibilities, reproduction in $D$. muliebre is primarily,

231 if not entirely, asexual.

In any case, to our knowledge, this study represents the first demonstration of accelerated

233 mutation accumulation in a putatively asexual organism that is apparently capable of some

234 genome-wide recombination. In a recent analysis comparing sexual and automictic asexual

235 Trichogramma pretiosum wasps, Lindsey et al. (2018) did not find increased rates of protein

236 evolution in asexuals; however, the absence of a sexual outgroup to T. pretiosum prohibited

237 genome-wide rate tests at the nucleotide level. Our comparisons across reproductive modes are

238 largely based on single individual wasps as proxies for entire lineages. Our whole-genome NGS

239 datasets from five previously-identified lineages of D. muliebre wasps exhibited very low

240 intraspecific variation (Table S4, Figure S3, Supplemental Information), such that our

241 conclusions about one asexual individual reasonably extend to the asexual species. However,

242 increased sampling from $D$. muliebre and D. ferrugineum wasps-for example to determine

243 ratios of nonsynonymous to synonymous changes that are fixed $v s$. polymorphic — will be needed

244 to draw further conclusions about genomic consequences of asexuality. Both of these ratios were 
245 higher in asexual lineages of Timema stick insects, demonstrating that putatively deleterious

246 variants are fixed faster and remain polymorphic for longer in asexuals (Bast et al. 2018).

248 evolution, a new proposition emerges from these data: Asexual animals derived from

249 haplodiploid ancestors - already proposed to be initially more successful (Maynard Smith

250 1978) - in the presence of automixis (and/or rare sex), may also be less vulnerable to the

251 genomic degradation that general theory suggests should plague other asexuals. Haplodiploid

252 arthropods, which are particularly rich in reproductive transitions to obligate asexuality (van der

253 Kooi et al. 2017), possess a wide variety of automictic mechanisms (Suomalainen et al. 1987;

254 Stenberg and Saura 2009). While transitions to asexual reproduction from diploid sexuals might

255 be generally disadvantageous due to frequent uncovering of deleterious recessive alleles, this

256 component of genetic load is likely to initially be much lower in haplodiploids (e.g. Diachasma),

257 where recessive harmful variants are fully expressed in males (Archetti 2004). Indeed, a recent in

258 silico study suggested that automictic asexual lineages could possess both greater genetic

259 diversity and lower genetic loads relative to sexual lineages (Engelstädter 2017).

260 Now consider an emerging asexual lineage with high diversity, low genetic load, and

261 maintenance of meiotic recombination (via automixis or rare sex): its risk of genome degradation

262 may be outweighed by its promise for adaptation. Classical hypotheses for the evolutionary

263 maintenance of sex invoke recombination's activity to bring together advantageous combinations

264 of alleles (Fisher 1930; Muller 1932) or to generate genetic diversity to increase occupancy of

265 heterogeneous niches (Bell 1982). Both of these advantages - typically reserved for sexual

266 organisms - may apply to D. muliebre, a haplodipoid asexual that maintains recombination and

267 has a measurable increase in molecular evolutionary rate as a source of new variation. Indeed, 
268 while sexual D. alloeum and D. ferrugineum exhibit relatively wide phenotypic variation for

269 adult emergence timing (Forbes et al. 2009) (a trait critical to wasp fitness; Supplemental

270 Information), individual lineages of $D$. muliebre have apportioned the temporal niche of their

271 sexual relatives: each has shorter, and incompletely overlapping, emergence windows, with some

272 lineages emerging relatively earlier and others relatively later (Forbes et al. 2013). Earlier-

273 emerging lineages of $D$. muliebre have also recently expanded into a new habitat niche following

274 environmental change (Forbes et al. 2013) (Supplemental Information). Access to different

275 combinations of genetic and phenotypic variation may thus reduce competition among asexual

276 lineages, equip an asexual to navigate new environments, and allow asexuals to persist for longer

277 than theory has previously suggested. 


\section{Acknowledgements}

279 We thank Amanda Nelson and Wee Yee for help with wasp collections, Gery Hehman for

280 assistance with Illumina library preparation, and Nick Stewart and Laura Bankers for preliminary

281 sequencing efforts of Diachasma wasps that motivated the study. We thank Maurine Neiman and

282 Bryant McAllister and anonymous reviewers of a previous version for their insightful comments

283 on the manuscript. This work was funded by a University of Iowa Internal Funding Initiative

284 grant to A.A.F. and J.M.L. and an NSF DEB (1145355) to AAF.

\section{Author contributions}

286 E.S.T., A.A.F., and J.M.L. conceptualized the study, designed sequencing experiments, and

287 wrote the manuscript. E.S.T and A.C.W. wrote custom scripts for analysis of sequencing data,

288 E.S.T. and B.T. performed analyses on sequencing data.

\section{Declaration of Interests}

290 The authors declare no competing interests.

\section{Data availability}

292 Illumina sequencing datasets will be made available on the NCBI SRA database (Table S2). 


\section{Materials and Methods}

\section{Preparation of Diachasma genomic libraries}

296 We obtained individual female and male Diachasma specimens for Illumina sequencing

297 extractions. All collections were made by first collecting Rhagoletis fly-infested fruits. Fly larvae

298 were allowed to emerge from fruits and pupate in the lab and were then artificially overwintered

299 in a refrigerator. Wasps emerged from some pupae the following year. We collected two $D$.

300 ferrugineum wasps (one female, one male) in Iowa City, IA, U.S.A. $\left(41.39^{\circ} \mathrm{N}, 91.31^{\circ} \mathrm{W}, 204 \mathrm{~m}\right.$

301 elevation), one D. ferrugineum male in South Bend, IN, U.S.A. (41.39 N, 91.31 ${ }^{\circ} \mathrm{W}, 204 \mathrm{~m}$

302 elevation) and five D. muliebre females in Roslyn, WA, U.S.A. $\left(47.22^{\circ} \mathrm{N}, 120.99^{\circ} \mathrm{W}, 685 \mathrm{~m}\right.$

303 elevation). The D. muliebre individuals were obtained from a larger collection of wasps and

304 represented five different previously-identified mitochondrial cytochrome C oxidase I (COX1)

305 haplotypes (21). We generated RNA libraries for the Iowa City samples using the TruSeq

306 Stranded mRNA kit (Illumina Inc., San Diego, CA) and DNA libraries for the remaining samples

307 using the KAPA Hyper Prep kit (KAPA Biosystems, Wilmington, MA). We sequenced paired-

308 end DNASeq and RNASeq reads $(2 \times 300)$ at the University of Iowa using the Illumina MiSeq

309 platform (Illumina Inc., San Diego, CA). After inspecting raw read data, we performed quality

310 trimming on reads using Trimmomatic v.0.32 (Bolger et al. 2014) and visually confirmed

311 trimmed datasets using FASTQC (Babraham Bioinformatics;

312 https://www.bioinformatics.babraham.ac.uk/projects/fastqc/).

\section{Reference guided genome assembly and variant calling}

314 Given the haplodiploid genetic system of Diachasma, we conducted SNP calling pipelines in the

315 nuclear genome with all female samples (one D. ferrugineum, five D. muliebre). An overview of 316 the Illumina dataset processing pipeline is provided in Figure S4. We adopted the GATK's Best 
317 Practices (Van der Auwera et al. 2013) for generating distinct pipelines for the processing of

318 DNASeq vs. RNASeq datasets (https://software.broadinstitute.org/gatk/best-practices/; accessed

319 2017).

320 There are three major differences in the RNA vs. DNA pipeline. First, we used different

321 programs to map trimmed reads to the $D$. alloeum reference genome. We mapped reads passing

322 quality filters to the D. alloeum genome assembly Dall1.0 (Tvedte et al. 2019b) (NCBI

323 Accession GCA_001412515.1). We mapped DNASeq reads using Bowtie2 v.2.3.0 (Langmead

324 and Salzberg 2012) and RNASeq reads using TopHat2 v.2.1.1 (Kim et al. 2013). To map reads

325 to the D. alloeum genome, we largely used the default parameters for these tools. For TopHat2,

326 we set the mean inner distance between mate pairs to $450 \mathrm{bp}$ (SD of $20 \mathrm{bp}$ ), and for Bowtie2, we

327 set the maximum fragment length for valid paired-end alignments to 800, as these values were

328 consistent with the Illumina library insert sizes. Using the 'genomecov' and 'maskfasta'

329 functions from BEDTools v.2.26.0 (Quinlan and Hall 2010), we generated new D. alloeum

330 reference sequences with masked regions that had $<2 \mathrm{X}$ coverage. Reads were then re-mapped to

331 the masked reference using Bowtie2 and TopHat2.

332 Second, for the RNASeq dataset, we executed the GATK program SplitNCigarReads,

333 prior to variant calling using HaplotypeCaller. This tool splits mapped reads containing Ns

334 corresponding to splice junctions and is appropriate only for RNA datasets.

Third, we used specific parameters to filter DNASeq and RNASeq variants using GATK

336 VariantFiltration. Assemblers use different systems for defining the mapping quality (MAPQ)

337 value of reads, and manual inspection of alignments indicated a single MAPQ filter value would

338 not work well across variant callsets. We excluded reads with $\mathrm{MQ}<2$ aligned with Bowtie2 and

339 excluded reads with MQ $<40$ aligned with TopHat2 and BWA-MEM. All other filtering 
parameters were identical for all datasets, namely: QD >2.0, FS >60.0, MQRankSum <-12.5,

341 ReadPosRankSum <-8.0, DP $>4$ ). After mapping, we manually inspected a subset of genes to

342 assess SNP calling accuracy (Table S5, Table S6, Supplemental Information).

\section{Ortholog identification and CDS dataset generation}

344 We downloaded protein datasets from the D. alloeum (Tvedte et al. 2019b), F. arisanus (Geib et 345 al. 2017), and M. demolitor (Burke et al. 2018) genome assemblies. Using a custom script, we 346 produced nonredundant protein datasets for each species. Protein datasets were submitted to

347 OrthoVenn v.1 (Wang et al. 2015) for ortholog identification. Using 7,910 single-copy gene 348 clusters assigned by OrthoVenn, we retained 3,127 with full CDS coverage in all wasp species 349 compared in this study, including individual D. ferrugineum and D. muliebre females. Using the

350 GFF file associated with each genome assembly, we ran a custom script to retrieve genome 351 coordinates for all CDS regions. For D. alloeum and F. arisanus, we provided CDS coordinates 352 as the $-\mathrm{L}$ parameter to retrieve CDS for each gene using GATK FastaReferenceMaker. For $D$. 353 ferrugineum and D. muliebre, we executed a similar strategy, using the appropriate masked 354 genome as the $-\mathrm{R}$ parameter and the VCF file from the GATK Best Practices pipeline as the $-\mathrm{V}$ 355 parameter using GATK FastaAlternateReferenceMaker to obtain CDS for these wasp species.

356 For each of the genes in the dataset, we tested whether the asexual lineage D. muliebre possesses 357 a greater mutational load relative to the sexual lineage, D. ferrugineum. To do this, we produced 358 codon-aware CDS alignments using PAL2NAL v.14 (Suyama et al. 2006), providing combined 359 information from MUSCLE protein alignments (Edgar 2004) and corresponding nucleotide 360 sequences. We retained only genes having full CDS coverage in all wasp species, and calculated

361 p-distances for each gene using the 'ape' package in R (Paradis et al. 2004). Specifically, we 362 measured p-distances for D. muliebre (pdistDM) and D. ferrugineum (pdistDF) relative to a 
363 common sexual outgroup, D. alloeum. Although p-distance values include evolutionary changes

364 that have occurred in D. alloeum and the stem lineage preceding the divergence of $D$. muliebre

365 and D. ferrugineum, using D. alloeum as a common reference point for comparisons allows us to

366 infer whether a greater number of mutational differences have occurred in the asexual or sexual

367 lineage. To achieve this, we first calculated the difference between pairwise distances (pdistDM-

368 pdistDF) for each gene, and subsequently conducted a chi-square analysis to test against a null

369 distribution of equal numbers of genes with greater mutational differences in sexuals and

$370 \quad$ asexuals $(($ pdistDM $>$ pdistDF $)=($ pdistDM $<$ pdistDF $))$.

371 Variation in library preparation, sequencing, and assembly methods might generate distinct

372 artefacts in our pipeline. To reduce the likelihood of problematic variant calling in Illumina

373 datasets, we manually inspected a subset of genes to assess whether GATK produces accurate

374 SNP calls across dataset types (i.e. DNASeq vs. RNASeq) (Supplemental Information). The

375 above analyses treat each gene as an independent unit, however the actual dataset might be

376 biased towards genes contained in linkage groups with nonindependent selective pressures. We

377 determined the assembly scaffolds represented by the genes in the dataset to assess potential

378 biases generated by linkage (Supplemental Information).

\section{Substitution rate analyses}

380 We compiled the codon-aware, pairwise alignments of each CDS between either $D$.

381 alloeum and D. muliebre or D. alloeum and D. ferrugineum into their own respective text files.

382 Next, we analyzed each of these files for codon substitutions using codeml in PAML v.4.8 (Yang

383 2007) with a two-branch tree containing D. alloeum and either D. muliebre or D. ferrugineum.

384 We calculated one $\omega$ ratio for all branches in the tree for each gene and calculated the average 
nucleotide frequency at all three codon positions, assuming no clock. We then grouped each

CDS by its synonymous $(\mathrm{dS})$ and non-synonymous $(\mathrm{dN})$ substitution rates.

\section{Relative rate analyses}

388 In addition to evaluating mutational patterns for each nuclear gene individually, we performed a

389 global analysis to characterize whether sex loss in Diachasma is associated with the

390 accumulation of deleterious mutations. We combined all CDS regions with full coverage into a

391 single concatenated dataset. Next, we used the GTR+CAT model implemented in RAxML

392 (Stamatakis 2006) to conduct a maximum likelihood analysis in order to estimate branch lengths

393 between sexual and asexual species. We performed three RAxML runs, using 1) all CDS sites, 2)

$3941^{\text {st }}+2^{\text {nd }}$ codon positions $(\approx$ nonsynonymous sites $)$, and 3$) 3^{\text {rd }}$ codon positions $(\approx$ synonymous

395 sites).

We performed an implementation of Tajima's Relative Rate test in MEGA6 (Tajima

397 1993; Tamura et al. 2013) to test for evolutionary rate differences in D. muliebre and D.

398 ferrugineum. The test requires a three-taxon sequence dataset with knowledge a priori of the

399 outgroup relative to the other two. Life history, morphology, and genetic data all support $D$.

400 ferrugineum as the closest sexual relative to $D$. muliebre, with $D$. alloeum as the outgroup

401 (Wharton and Marsh 1978; Forbes et al. 2009; Hamerlinck et al. 2016). We calculated the

402 number of observed unique SNPs in the concatenated dataset for each Diachasma species. Next,

403 a chi-square test compares the observed number of unique differences to the expected number,

404 given equal evolutionary rates. We performed three separate analyses to test rate differences

405 across all sites as well as approximate nonsynonymous $\left(1^{\text {st }}+2^{\text {nd }}\right.$ codon positions $)$ and

406 synonymous ( $3^{\text {rd }}$ codon positions) sites. To be conservative, we applied the $D$. muliebre false-

407 positives rate to the concatenated dataset to compare wasp genomes (the application of the false 
negatives rate as a proportion of true negatives in this case is negligible given the total length of

409 the alignments). If $\sim 108(1.89 \%)$ D. muliebre SNPs are false positives, this individual would

410 have 5,634 SNPs in the concatenated dataset. Applying this conservative measure, the number of

411 unique differences was significantly greater than the 4,148 observed in D. ferrugineum $\left(\chi^{2}=\right.$

$4121416, \mathrm{p}<0.005)$

\section{Intraspecific comparisons}

414 To assess whether there is substantial variation in the nuclear genome mutation load among

415 individual asexual females, we sequenced DNASeq datasets from five $D$. muliebre females.

416 Applying the methods described above, we retrieved a subset of the conserved genes that had

417 full-length coverage in the single D. ferrugineum female and all D. muliebre females. After

418 calculating pairwise distances for each wasp relative to D. alloeum, we performed multiple t-

419 tests to test for differences among the asexual distributions, applying a Bonferroni correction for

420 multiple comparisons.

\section{Codon usage bias and gBGC}

422 We conducted two separate tests to evaluate differences in genome-wide codon usage between

423 D. ferrugineum and D. muliebre females. First, we determined the effective number of codons

424 (ENC) for each gene (Wright 1990). The range of ENC values reflect codon usage for each

425 amino acid, from 20 (each amino acid encoded by one codon) to 61 (all codons used equally).

426 We calculated ENC using the program ENCprime (https://github.com/jnovembre/ENCprime),

427 which accounts for background nucleotide compositions to enable cross-species comparisons

428 (Novembre 2002). Second, we used the Composition Analysis Toolkit (CAT;

429 http://www.cbrc.kaust.edu.sa/CAT) to calculate per-gene codon deviation coefficient (CDC)

430 values (Zhang et al. 2012). CAT uses background GC and purine content for each codon position 
431 to calculate an expected codon usage value and calculates CDC as a deviation from this

432 expectation. CDC values range from 0 (no deviation from expectation, i.e. no selection on codon

433 usage) to 1 (maximum deviation, i.e. effective purifying selection). To assess gBGC, we used

434 CAT to calculate per-gene GC content at third codon positions (GC3), which are most likely to

435 be evolving neutrally. Higher GC3 values reflect preferential conversion of A and T alleles to G

436 and $\mathrm{C}$.

\section{Statistical analyses}

439 For comparisons of pairwise distances, ENC, CDC, and GC3 in the genome, we performed

440 statistical tests to determine whether the distribution of values involving $D$. muliebre and $D$.

441 ferrugineum were different. We tested each dataset for normality using the Shapiro-Wilk

442 method. Since all tests failed tests of normality (Table S8), we conducted a Wilcoxon rank sum

443 test as a conservative measure to determine whether or not sexual and asexual wasps possess

444 similar pairwise distance distributions.

\section{Supplemental Information}

447 Supplementary Material. Figures S1 - S5. Tables S1 - S8. 
448

449

450

451

452

453

454

455

456

457

458

459

460

461

462

463

464

465

466

467

468

469

470

\section{References}

Ament-Velásquez, S. L., E. Figuet, M. Ballenghien, E. E. Zattara, J. L. Norenburg, F. A. Fernández-Álvarez, J. Bierne, N. Bierne, and N. Galtier. 2016. Population genomics of sexual and asexual lineages in fissiparous ribbon worms (Lineus, Nemertea): hybridization, polyploidy and the Meselson effect. Molecular Ecology 25:3356-3369.

Archetti, M. 2004. Loss of complementation and the logic of two-step meiosis. Journal of Evolutionary Biology 17:1098-1105.

Bast, J., D. J. Parker, Z. Dumas, K. M. Jalvingh, P. Tran Van, K. S. Jaron, E. Figuet, A. Brandt, N. Galtier, and T. Schwander. 2018. Consequences of asexuality in natural populations: insights from stick insects. Molecular Biology and Evolution 35:1668-1677.

Bell, G. 1982. The Masterpiece of Nature: The Evolution and Genetics of Sexuality. Cambridge University Press, Cambridge, UK.

Bolger, A. M., M. Lohse, and B. Usadel. 2014. Trimmomatic: a flexible trimmer for Illumina sequence data. Bioinformatics 30:2114-2120.

Brandt, A., J. Bast, S. Scheu, K. Meusemann, A. Donath, K. Schütte, R. Machida, and K. Kraaijeveld. 2019. No signal of deleterious mutation accumulation in conserved gene sequences of extant asexual hexapods. Scientific Reports 9:5338.

Brandt, A., I. Schaefer, J. Glanz, T. Schwander, M. Maraun, S. Scheu, and J. Bast. 2017. Effective purifying selection in ancient asexual oribatid mites. Nature Communications 8:873.

Burke, G. R., K. K. O. Walden, J. B. Whitfield, H. M. Robertson, and M. R. Strand. 2018. Whole genome sequence of the parasitoid wasp Microplitis demolitor that harbors an endogenous virus mutualist. G3: Genes|Genomes|Genetics 8:2875. 
471 Charlesworth, B. and D. Charlesworth. 1997. Rapid fixation of deleterious alleles can be caused by Muller's ratchet. Genetics Research 70:63-73.

473 Edgar, R. C. 2004. MUSCLE: multiple sequence alignment with high accuracy and high throughput. Nucleic Acids Research 32:1792-1797.

Engelstädter, J. 2017. Asexual but not clonal: evolutionary processes in automictic populations. Genetics 206:993.

477 Felsenstein, J. 1974. The evolutionary advantage of recombination. Genetics 78:737-756.

478 Fisher, R. 1930. The Genetical Theory of Natural Selection. Oxford: Clarendon Press. 272 p.

479 Forbes, A. A., T. H. Q. Powell, L. L. Stelinski, J. J. Smith, and J. L. Feder. 2009. Sequential sympatric speciation across trophic levels. Science 323:776.

481 Forbes, A. A., L. A. Rice, N. B. Stewart, W. L. Yee, and M. Neiman. 2013. Niche differentiation and colonization of a novel environment by an asexual parasitic wasp. Journal of Evolutionary Biology 26:1330-1340.

Geib, S. M., G. H. Liang, T. D. Murphy, and S. B. Sim. 2017. Whole genome sequencing of the braconid parasitoid wasp Fopius arisanus, an important biocontrol agent of pest Tepritid

Glémin, S. and N. Galtier. 2012. Genome evolution in outcrossing versus selfing versus asexual Species. Pp. 311-335 in M. Anisimova, ed. Evolutionary Genomics: Statistical and Computational Methods, Volume 1. Humana Press, Totowa, NJ. 
493

494

495

496

497

498

499

500

501

502

503

504

505

506

507

508

509

510

511

512

513

514

515

Hartfield, M. 2016. Evolutionary genetic consequences of facultative sex and outcrossing. Journal of Evolutionary Biology 29:5-22.

Hartfield, M. and P. D. Keightley. 2012. Current hypotheses for the evolution of sex and recombination. Integrative Zoology 7:192-209.

Hill, W. G. and A. Robertson. 1966. The effect of linkage on limits to artificial selection. Genetics Research 8:269-294.

Hollister, J. D., S. Greiner, W. Wang, J. Wang, Y. Zhang, G. K.-S. Wong, S. I. Wright, and M. T. J. Johnson. 2014. Recurrent loss of sex is associated with accumulation of deleterious mutations in Oenothera. Molecular Biology and Evolution 32:896-905.

Jaron, K. S., J. Bast, R. W. Nowell, T. R. Ranallo-Benavidez, M. Robinson-Rechavi, and T. Schwander. 2020. Genomic features of parthenogenetic animals. bioRxiv:497495.

Kim, D., G. Pertea, C. Trapnell, H. Pimentel, R. Kelley, and S. L. Salzberg. 2013. TopHat2: accurate alignment of transcriptomes in the presence of insertions, deletions and gene fusions. Genome Biology 14:R36.

Langmead, B. and S. L. Salzberg. 2012. Fast gapped-read alignment with Bowtie 2. Nature Methods 9:357.

Lindsey, A. R. I., Y. D. Kelkar, X. Wu, D. Sun, E. O. Martinson, Z. Yan, P. F. Rugman-Jones, D. S. T. Hughes, S. C. Murali, J. Qu, S. Dugan, S. L. Lee, H. Chao, H. Dinh, Y. Han, H. V. Doddapaneni, K. C. Worley, D. M. Muzny, G. Ye, R. A. Gibbs, S. Richards, S. V. Yi, R. Stouthamer, and J. H. Werren. 2018. Comparative genomics of the miniature wasp and pest control agent Trichogramma pretiosum. BMC Biology 16:54.

Lovell, J. T., R. J. Williamson, S. I. Wright, J. K. McKay, and T. F. Sharbel. 2017. Mutation accumulation in an asexual relative of Arabidopsis. PLOS Genetics 13:e1006550. 
Lynch, M., R. Bürger, D. Butcher, and W. Gabriel. 1993. The Mutational Meltdown in Asexual Populations. Journal of Heredity 84:339-344.

518 Maynard Smith, J. 1978. The evolution of sex. Cambridge University Press Cambridge.

519 Muller, H. J. 1932. Some genetic aspects of sex. The American Naturalist 66:118-138.

520 Muller, H. J. 1964. The relation of recombination to mutational advance. Mutation

521 Research/Fundamental and Molecular Mechanisms of Mutagenesis 1:2-9.

522 Novembre, J. A. 2002. Accounting for background nucleotide composition when measuring codon usage bias. Molecular Biology and Evolution 19:1390-1394.

524 Otto, Sarah P. 2009. The evolutionary enigma of sex. The American Naturalist 174:S1-S14.

525 Paradis, E., J. Claude, and K. Strimmer. 2004. APE: Analyses of Phylogenetics and Evolution in R language. Bioinformatics 20:289-290.

527 Quinlan, A. R. and I. M. Hall. 2010. BEDTools: a flexible suite of utilities for comparing genomic features. Bioinformatics 26:841-842.

529 Schurko, A. M., M. Neiman, and J. M. Logsdon. 2009. Signs of sex: what we know and how we know it. Trends in Ecology \& Evolution 24:208-217.

531 Smith, R. J., T. Kamiya, and D. J. Horne. 2006. Living males of the 'ancient asexual' Darwinulidae (Ostracoda: Crustacea). Proceedings of the Royal Society B: Biological Sciences 273:1569-1578.

534 Stamatakis, A. 2006. RAxML-VI-HPC: maximum likelihood-based phylogenetic analyses with thousands of taxa and mixed models. Bioinformatics 22:2688-2690.

536 Stenberg, P. and A. Saura. 2009. Cytology of asexual animals. Pp. 63-74 in I. Schön, K. Martens, and P. Dijk, eds. Lost Sex: The Evolutionary Biology of Parthenogenesis. Springer

$538 \quad$ Netherlands, Dordrecht. 
Suomalainen, E., A. Saura, and J. Lokki. 1987. Cytology and Evolution in Parthenogenesis. CRC Press.

541 Suyama, M., D. Torrents, and P. Bork. 2006. PAL2NAL: robust conversion of protein sequence alignments into the corresponding codon alignments. Nucleic Acids Research 34:W609-

Tajima, F. 1993. Simple methods for testing the molecular evolutionary clock hypothesis. W612. Genetics 135:599-607.

Tvedte, E. S., A. A. Forbes, and J. M. Logsdon, Jr. 2017. Retention of core meiotic genes across diverse Hymenoptera. Journal of Heredity 108:791-806.

Tamura, K., G. Stecher, D. Peterson, A. Filipski, and S. Kumar. 2013. MEGA6: Molecular Evolutionary Genetics Analysis Version 6.0. Molecular Biology and Evolution 30:2725-

Tvedte, E. S., K. K. O. Walden, K. E. McElroy, J. H. Werren, A. A. Forbes, G. R. Hood, J. M. Logsdon, Jr, J. L. Feder, and H. M. Robertson. 2019b. Genome of the parasitoid wasp Diachasma alloeum, an emerging model for ecological speciation and transitions to asexual reproduction. Genome Biology and Evolution 11:2767-2773.

557 Van der Auwera, G. A., M. O. Carneiro, C. Hartl, R. Poplin, G. del Angel, A. Levy-Moonshine, T. Jordan, K. Shakir, D. Roazen, J. Thibault, E. Banks, K. V. Garimella, D. Altshuler, S. Gabriel, and M. A. DePristo. 2013. From FASTQ data to high-confidence variant calls: the Genome Analysis Toolkit best practices pipeline. Current Protocols in Bioinformatics 43:11.10.11-11.10.33. 
562 van der Kooi, C. J., C. Matthey-Doret, and T. Schwander. 2017. Evolution and comparative

563 ecology of parthenogenesis in haplodiploid arthropods. Evolution letters 1:304-316.

564 Wang, Y., D. Coleman-Derr, G. Chen, and Y. Q. Gu. 2015. OrthoVenn: a web server for genome

565 wide comparison and annotation of orthologous clusters across multiple species. Nucleic

$566 \quad$ Acids Research 43:W78-W84.

567 Wharton, R. A. and P. M. Marsh. 1978. New world opiinae (hymenoptera: Braconidae) parasitic on tephritidae (diptera). Journal of the Washington Academy of Sciences:147-167.

569 Wright, F. 1990. The 'effective number of codons' used in a gene. Gene 87:23-29.

570 Yang, Z. 2007. PAML 4: Phylogenetic Analysis by Maximum Likelihood. Molecular Biology and Evolution 24:1586-1591.

572 Zhang, Z., J. Li, P. Cui, F. Ding, A. Li, J. P. Townsend, and J. Yu. 2012. Codon Deviation

573 Coefficient: a novel measure for estimating codon usage bias and its statistical

$574 \quad$ significance. BMC Bioinformatics 13:43. 


\section{Figure Legends}

577 Figure 1. Relative pairwise distances in sexual and asexual Diachasma.

578 Positive (red) values indicate genes with more nucleotide differences in asexuals (pdistDM >

579 pdistDF), negative (blue) values indicate genes with more differences in sexuals (pdistDM $<$

580 pdistDF), zero (purple) values indicate genes with equivalent distances (pdistDM $=$ pdistDF).

581 Bolded black lines correspond to the 90th percentile of positive and negative $\Delta$ values. All

582 pairwise distance values were calculated against a common wasp reference (D. alloeum).

583 Figure 2. Mutation landscapes of sexual and asexual Diachasma.

584 Columns represent binned frequencies (left y-axis) of corresponding p-distance values from $D$.

585 alloeum. Curves represent the cumulative frequency of binned columns (right y-axis).

Figure 3. Maximum likelihood analysis of concatenated nuclear gene dataset in Diachasma.

587 Asexual (red) and sexual (blue) Diachasma lineages are shown, and informative branch lengths

588 are labeled. A. Phylogenetic tree of all sites $(3,715,368 \mathrm{bp})$. B. Phylogenetic tree of $1^{\text {st }} / 2^{\text {nd }}$ codon

589 sites $\left(2,476,912\right.$ bp). C. Phylogenetic tree of $3^{\text {rd }}$ codon sites $(1,238,456$ bp).

590 Figure 4. Comparison of replacement substitutions (dN) in 3,127 orthologs from $D$.

$591 \quad$ muliebre and $D$. ferrigineum, compared to D. alloeum.

592 Replacement substitutions $(\mathrm{dN})$ were calculated in PAML comparing D. muliebre and D.

593 ferrigineum to $D$. alloeum in three species alignments. The total number of genes in each bin for

594 each species is shown; for example, there are 1346 and 1477 genes with $\mathrm{dN}=0-0.001$ (all with

595 identical amino acid sequences observed) in D.m. and D.f., respectively. For each bin, the

596 percent excess (or deficit) in D. muliebre is shown as a percentage compared to the number of 
bioRxiv preprint doi: https://doi.org/10.1101/2020.12.23.424202; this version posted December 24, 2020. The copyright holder for this preprint (which was not certified by peer review) is the author/funder. All rights reserved. No reuse allowed without permission.

597 genes for each class observed in D. muliebre. The bins marked with asterisks $\left({ }^{*}\right)$ are significantly

598 different (see text).

599 


\section{$600 \quad$ Figures}

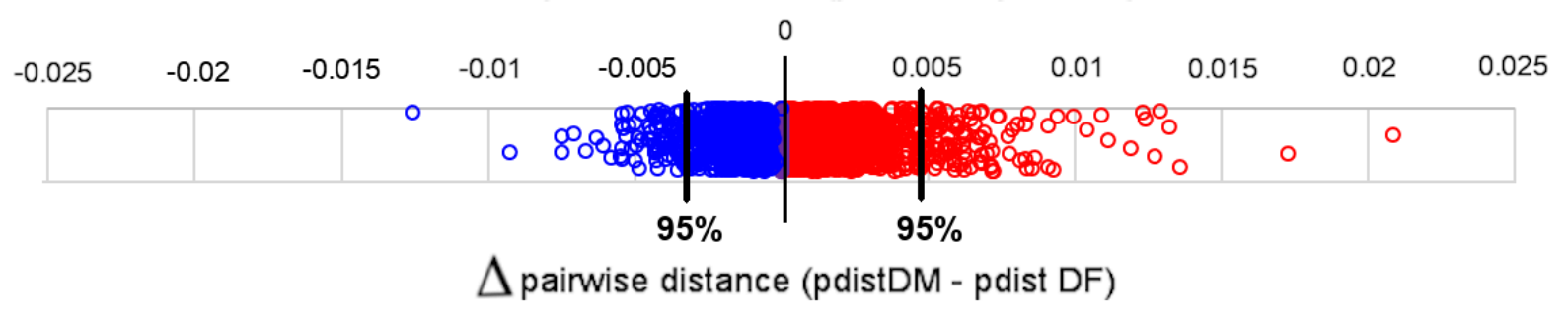

601

602 Figure 1. Relative pairwise distances in sexual and asexual Diachasma. 


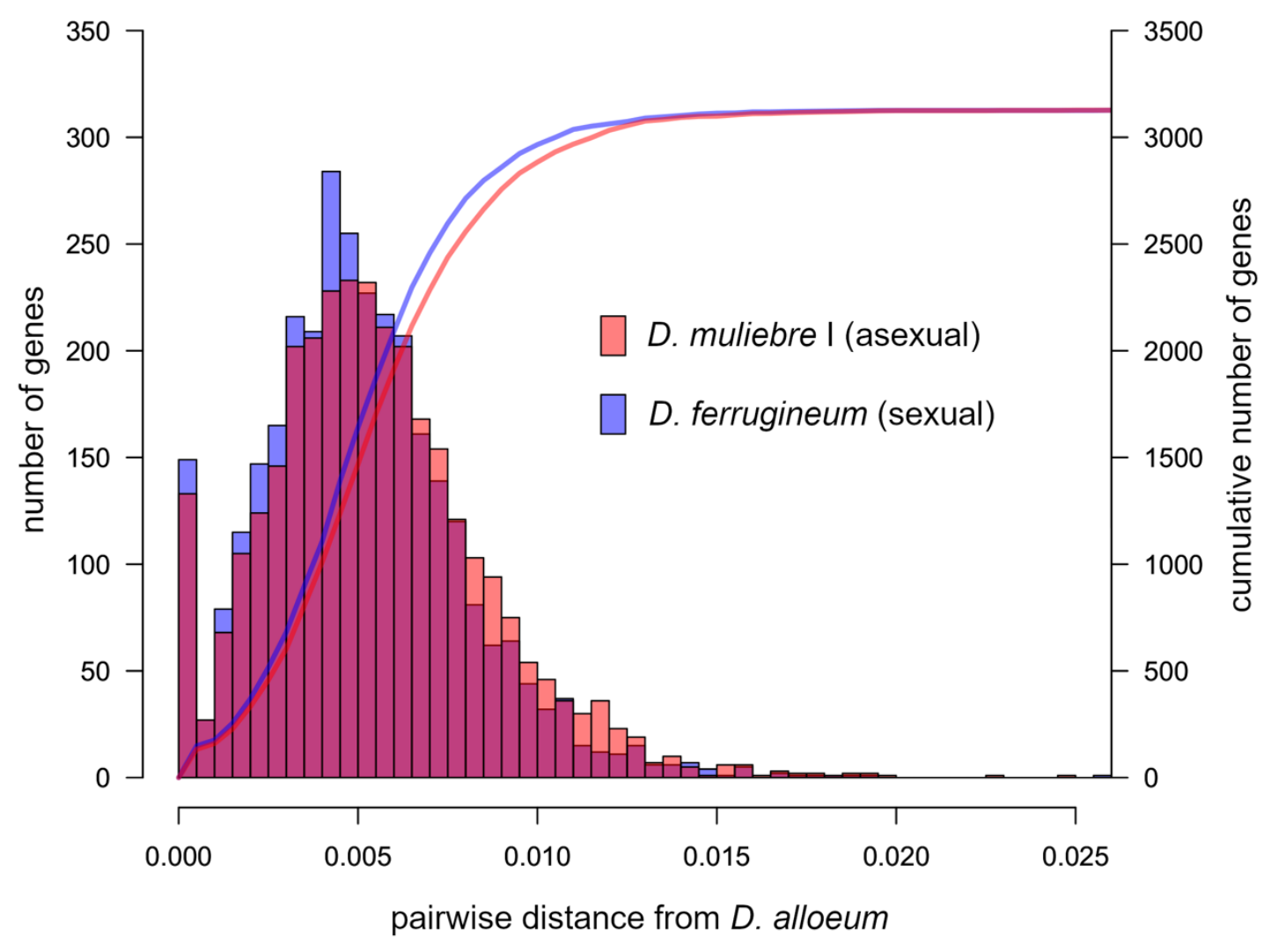

604 Figure 2. Mutation landscapes of sexual and asexual Diachasma. 

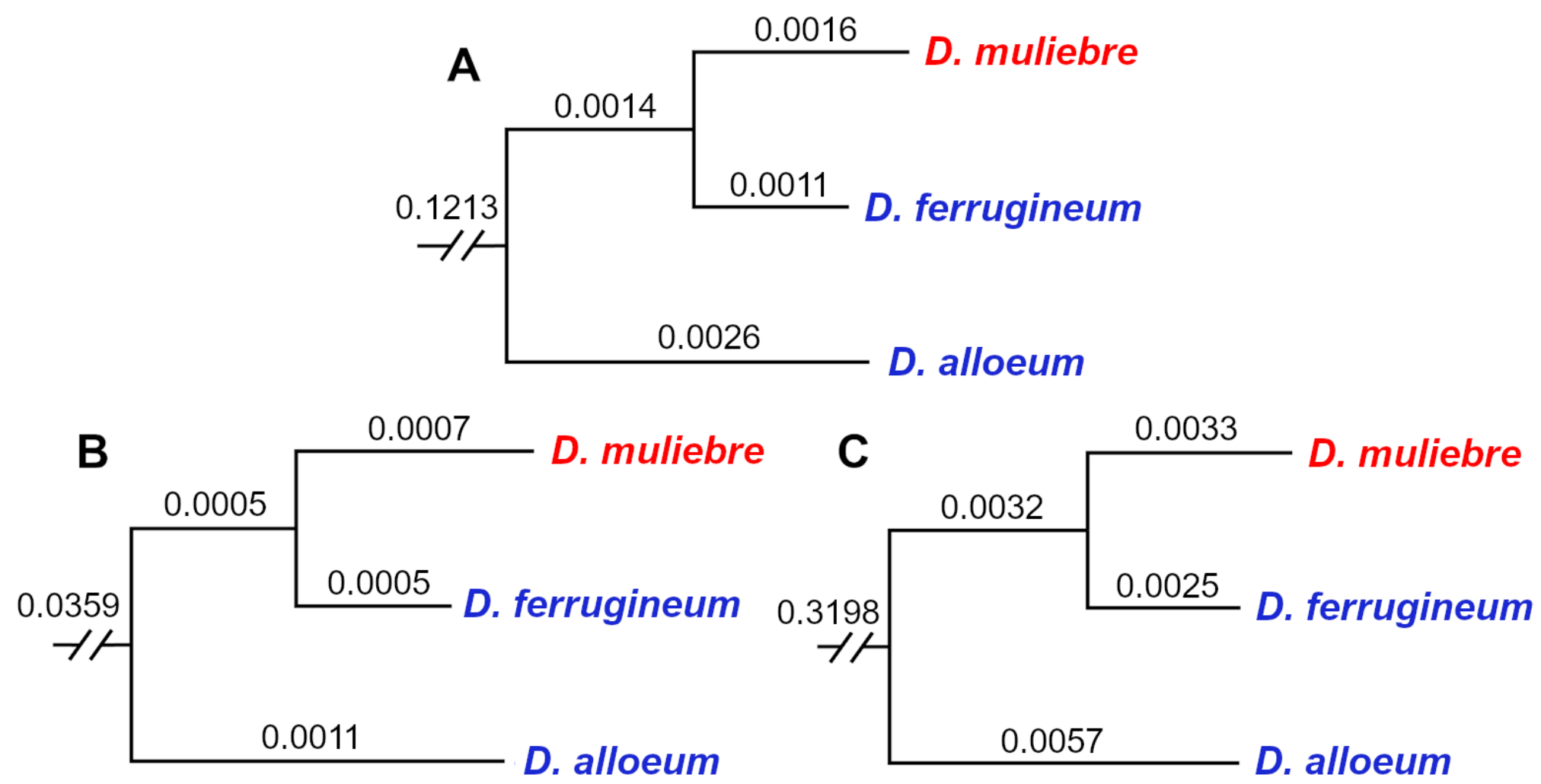

606

607 Figure 3. Maximum likelihood analysis of concatenated nuclear gene dataset in Diachasma 


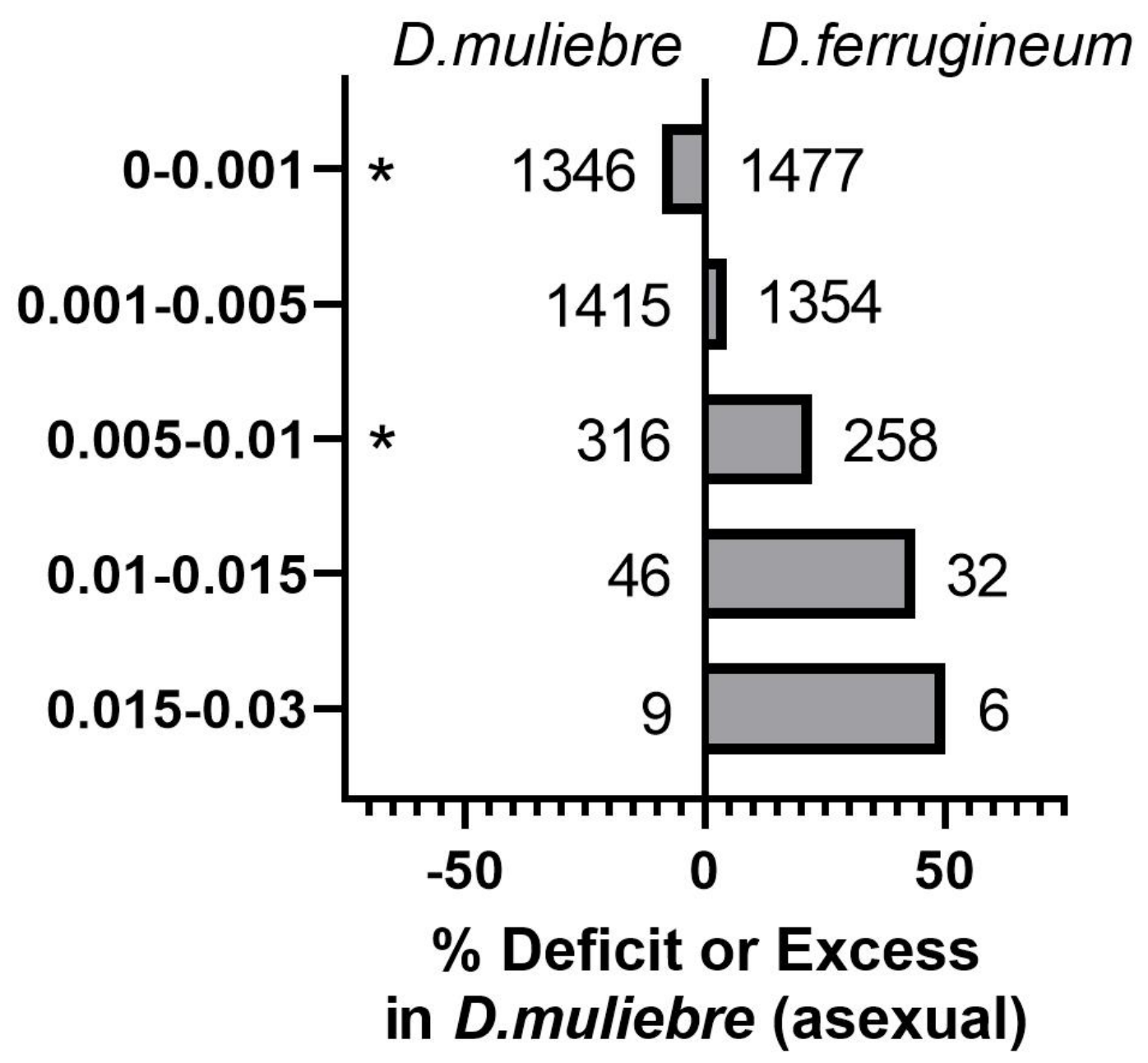

609

$610 \quad$ Figure 4. Comparison of replacement substitutions (dN) in 3127 orthologs from $D$.

611 muliebre and D. ferrigineum, compared to D. alloeum. 


\section{Tables}

613 Table 1. Relative rate analysis of concatenated gene set in Diachasma.

\begin{tabular}{|c|c|c|c|c|c|c|c|}
\hline & \multirow{2}{*}{\multicolumn{6}{|c|}{ number of unique differences }} \\
\hline & & & & & & & \\
\hline & & \multicolumn{2}{|c|}{ all sites } & \multicolumn{2}{|c|}{$\begin{array}{c}1^{\text {st }} / 2^{\text {nd }} \text { codon } \\
\text { positions }\end{array}$} & \multicolumn{2}{|c|}{$3^{\text {rd }}$ codon positions } \\
\hline $\begin{array}{c}\# \\
\text { genes }\end{array}$ & $\begin{array}{l}\text { length } \\
\text { (bp) }\end{array}$ & DF & DM & DF & DM & DF & $\mathrm{DM}$ \\
\hline 3,127 & $3,715,368$ & 4,148 & $5,742^{\mathrm{a}}$ & $\begin{array}{c}1,113 \\
(26.83 \%)\end{array}$ & $\begin{array}{c}1,702^{\mathrm{a}} \\
(29.64 \%)\end{array}$ & $\begin{array}{c}3,035 \\
(73.17 \%)\end{array}$ & $\begin{array}{c}\mathbf{4 , 0 4 0}{ }^{\mathrm{a}} \\
(70.36 \%)\end{array}$ \\
\hline
\end{tabular}

614 D. alloeum was used as the outgroup to measure the number of unique differences in the

615 sexual D. ferrugineum (DF) vs. asexual D. muliebre (DM) lineages. 14,598 sites were

616 shared by both DM and DF and 36 sites were unique in all three species. ${ }^{\mathrm{a}} \chi^{2}$ test statistic

617 was statistically significant $(\mathrm{p}<<0.005)$, i.e. the bolded value represents a significantly

618 higher number of unique differences than expected under a null model of equal

619 evolutionary rates. 